\title{
COMPLEXIDADES DO TRABALHO DO AGENTE COMUNITÁRIO DE SAÚDE COM PACIENTES EM USO DE MEDICAMENTOS
}

\author{
COMMUNITY HEALTH AGENTS AND THE COMPLEXITIES OF \\ THE WORKING WITH PATIENTS USING MEDICINES
}

\author{
COMPLEJIDAD DEL TRABAJO DEL AGENTE COMUNITÁRIO DE \\ SALUD CON PACIENTES EN USO DE MEDICAMENTOS
}

\author{
Maria do Carmo Vilas Boas Sousa ${ }^{1}$ \\ Cynthia Pimenta dos Santos ${ }^{2}$ \\ Simone de Araújo Medina Mendonça ${ }^{3}$
}

Resumo Os agentes comunitários de saúde representam importante elemento na gestão de informações sobre o uso de medicamentos na atenção primária, melhorando a interação da equipe de saúde com a comunidade. O objetivo do estudo foi revelar como o uso de medicamentos está presente na rotina deles e compreender as relações que se estabelecem entre agentes, usuários e equipe. Tratou-se de um estudo qualiquantitativo, com utilização de observação participante, entrevista semiestruturada e grupo focal. Foi desenvolvido em três unidades básicas de saúde em um município de Minas Gerais no período de março de 2013 a fevereiro de 2015. Os resultados revelaram que os agentes se relacionam constantemente com usuários que vivenciam problemas com o uso de medicamentos e apresentam dúvidas sobre suas indicações, efeitos esperados, reações adversas, entre outras. Eles realizam algumas intervenções diretamente com o usuário, porém preferem encaminhar os casos à equipe de saúde. Alguns agentes referiram-se ao farmacêutico como profissional de referência no tocante ao uso de medicamentos, mas a procura por esse profissional mostrou-se baixa pelos sujeitos do estudo. Ressalta-se a importância da formação do agente sobre utilização de medicamentos para instrumentalizá-los no reconhecimento de situações inadequadas, no desenvolvimento de intervenções com a equipe de saúde e no acompanhamento dos usuários. Palavras-chave uso de medicamentos; agente comunitário de saúde; pesquisa qualitativa.
Abstract The community health agents are an important element in the management of medication information in primary healthcare setting, improving healthcare team interaction with the community. The aim of this study was to reveal how the use of medicines is present in the routine of agentes and understand the relationships established between agents, users and healthcare team. This is a quantitative and qualitative study, using participant observation, semi-structured interview and focus group. This study was conducted at three basic health units in a municipality of Minas Gerais, Brazil, between March, 2013 and February, 2015. The results show that agents constantly relate with patients who experience drug therapy problems and have doubts about the indication, effects, adverse drug reactions, among other. They perform some interventions directly with the patient, but usually they prefer to refer cases to the healthcare team. Some agents said recognizing the pharmacist as a reference on medicines, but the demand for this professional was low by the subjects of this study. We emphasize the importance of the agents training on medication use to instrumentalize them to recognize problematic situations, to develop interventions with the support of the healthcare team and to follow up patients using medicines.

Keyswords drug therapy; community health agents; qualitative study. 


\section{Introdução}

O agente comunitário de saúde (ACS) fortalece a integração entre os serviços de atenção primária à saúde (APS) e a comunidade. Constitui-se elo entre equipe de saúde e usuários, relacionando o saber popular e o conhecimento técnico-científico (Marodin et al., 2011; Nunes, Amador e Heineck, 2008). Por ter amplo contato com a comunidade, conhecer e compartilhar a mesma cultura e, ao mesmo tempo, estar integrado à equipe de saúde, suas ações apresentam grande potencial para melhorar a qualidade de vida de indivíduos, famílias e comunidades (Brasil, 2006, 2009; Brito, Ferreira e Santos, 2014; Collinsworth et al., 2014; Costa et al., 2013; Kauling et al., 2013).

Vários estudos apontam para a relevância do trabalho dos ACSs quando integrados com as equipes multiprofissionais, auxiliando na melhoria do bem-estar da comunidade e na consolidação de mudanças nos sistemas de saúde. Balcazar e colaboradores (2011) destacam a efetividade da atuação do ACS por meio da gestão de doenças crônicas, uma vez que ele contribui para o empoderamento e o autocuidado do usuário. Um estudo realizado com 328 adultos hispânicos avaliou a efetividade de ações educativas sobre práticas de vida saudável e observou que os participantes acompanhados pelos ACSs apresentaram melhoria nos hábitos de saúde (Heer et al., 2012). Krantz e colaboradores (2013) demonstraram que o seguimento realizado pelo ACS tem impacto positivo na saúde dos indivíduos com hipertensão e outras condições crônicas, ao auxiliar na redução de fatores de risco para o desenvolvimento de doença cardíaca coronariana, como controle glicêmico e pressão arterial. Outro estudo, realizado com 164 afro-americanos e latinos com diabetes tipo 2, demonstrou uma redução dos níveis de HbAlc em usuários conduzidos pelos ACSs, em comparação com aqueles que não tiveram o acompanhamento (Spencer et al., 2011). Tais estudos apresentam a capacidade dos agentes em motivar mudanças de comportamento em populações de risco.

Ao se considerar o grande número de usuários crônicos de medicamentos na APS, o agente comunitário pode ser um elemento fundamental na gestão de informações seguras e na educação dos usuários sobre o uso correto de medicamentos (Goulart et al., 2014; Mastroianni et al., 2011; Medeiros et al., 2011). Alguns estudos ressaltam a importância do papel dos ACSs no acompanhamento dos usuários de medicamentos, uma vez que são capazes de identificar possíveis problemas com o uso inadequado devido à maior proximidade e interação com esses usuários. No estudo de Kauling e colaboradores (2013), foram destacados alguns problemas com medicamentos relacionados ao armazenamento, à automedicação, à posologia, às interações medicamentosas e reações adversas, entre outros. As autoras ressaltam as ações desenvolvidas pelos ACSs ante esses problemas e a importância de capacitação sobre o 
tema. Nunes, Amador e Heineck (2008) mostram a relevância do trabalho do ACS no auxílio à população quanto ao uso correto dos medicamentos, já que quando capacitado ele pode colaborar com a conscientização e a garantia do uso adequado. Em ambos os trabalhos, foi revelado que esse profissional não está preparado para atender às demandas dos indivíduos sobre seu tratamento medicamentoso.

As ações dos ACSs podem minimizar os problemas advindos da dificuldade na troca de conhecimentos sobre o uso de medicamentos entre usuários e profissionais de saúde. Para tanto, é necessário que eles estejam preparados para auxiliar o usuário a romper as barreiras que possam surgir quando as orientações recebidas forem insuficientes ou não realizadas pelos prescritores e farmacêuticos (Kauling et al., 2013; Laste et al., 2012; Marodin et al., 2011; Nunes, Amador e Heineck, 2008). Assim, destaca-se que são essenciais a formação e a educação permanente dos ACSs no campo da utilização de medicamentos.

O estudo aqui apresentado teve como objetivo descrever como o uso de medicamentos está presente na rotina dos ACSs e compreender as relações que se estabelecem entre eles, usuários e equipe de saúde na resolução de problemas identificados.

\section{Percurso metodológico}

O estudo foi realizado em três unidades básicas de saúde de um município na macrorregião centro-sul de Minas Gerais. Envolveu 26 ACSs que receberam capacitação sobre utilização de medicamentos, ofertada pela farmacêutica da equipe de apoio matricial em conjunto com uma farmacêutica residente, ambas da equipe da pesquisa aqui apresentada. Os agentes foram convidados e consentiram em participar do estudo de forma livre esclarecida.

Tratou-se de um estudo qualiquantitativo, com utilização de observação participante, aplicação de questionário semiestruturado e realização de grupo focal. Foi desenvolvido nas três unidades básicas de saúde no período de março/2013 a fevereiro/2015 por uma das pesquisadoras, que desempenhava também o papel de residente no programa de residência multiprofissional em atenção primária à saúde do município. Esse método permitiu conhecer a rotina de trabalho dos ACSs e sua relação com a equipe de saúde. Os dados foram registrados em diário de campo e posteriormente analisados para a construção dos resultados qualitativos; contribuíram também para a elaboração do questionário semiestruturado utilizado posteriormente para coleta de dados.

O questionário semiestruturado contemplou questões como frequência e principais dúvidas apresentadas aos ACSs pelos indivíduos que utilizam medicamentos e as condutas dos agentes ante esses questionamentos. Baseou-se 
o questionário na análise dos dados da observação participante e no estudo realizado por Marodin e colaboradores (2011). O questionário foi respondido diretamente pelos 26 ACSs participantes.

O grupo focal teve a participação de oito ACSs. Foi realizado para aprofundar a coleta de dados sobre os temas investigados com a pesquisa quantitativa e melhorar a compreensão de questões relacionadas ao uso de medicamentos nas atividades do ACS. Contou com duas pesquisadoras como moderadoras, que utilizaram um tópico guia para conduzir os sujeitos da pesquisa durante a coleta de dados. Os dados foram gravados, posteriormente transcritos e organizados para análise temática.

A pesquisa foi aprovada pelos comitês de ética em pesquisa envolvendo seres humanos do Hospital Municipal Odilon Behrens e da Secretaria Municipal de Saúde de Belo Horizonte, Minas Gerais, sob os protocolos de aprovação n. 826.446 e n. 862.394, respectivamente, e não há conflitos de interesse. Para manter o anonimato dos participantes, os ACSs foram identificados por números arábicos, e o áudio gravado durante o grupo focal foi destruído após transcrição e análise dos dados.

\section{Dificuldades enfrentadas pelos usuários com os medicamentos e condutas tomadas pelos ACSs}

As 26 ACSs que participaram do estudo são do sexo feminino, com média de idade de 43 anos. O tempo médio de trabalho como agente comunitário foi de $9,73 \mathrm{DP}=3,63$ anos, variando de 2 a 14 anos. Cada agente acompanha cerca de 250 famílias, o que corresponde a aproximadamente 750 pessoas por ACS. O maior número de pessoas acompanhadas por ACS foi 1.120 .

\section{O uso de medicamentos na rotina das ACSs}

Para 38,46\% $(\mathrm{n}=10)$ das ACSs, as dúvidas sobre o uso de medicamentos apareciam diariamente em algumas visitas domiciliares. Desse total, 26,93\% $(\mathrm{n}=10)$ relataram que as dúvidas eram apresentadas mensalmente em algumas visitas; já para 23,08\% $(\mathrm{n}=6)$, as dúvidas surgiam diariamente e na maioria das visitas; e para apenas $11,53 \%(n=3)$, semanalmente em algumas visitas ou nunca.

Segundo 38,46\% $(\mathrm{n}=10)$ das ACSs, a principal dúvida dos usuários era a indicação do uso do medicamento, seguindo-se os efeitos esperados - 23,08\% $(\mathrm{n}=6)$ - e reações adversas - 23,08\% $(\mathrm{n}=6)$ (Tabela 1). Os itens 'armazenamento', 'interação com alimentos' e 'formas de acesso aos medicamentos no SUS', embora constassem do instrumento de coleta de dados, não foram apontados pelas ACSs como dúvidas que os usuários lhes apresentassem no seu cotidiano. 
Tabela 1

\begin{tabular}{lc}
\hline Frequência dos tipos de dúvidas sobre medicamentos apresentadas às agentes comunitários em saúde \\
\hline $\begin{array}{l}\text { Tipos de dúvidas sobre } \\
\text { medicamentos apresentadas }\end{array}$ & $\%$ (n) \\
às ACSs pelos usuários & $38,46 \%(10)$ \\
\hline Indicação de uso & $23,08 \%(6)$ \\
Efeitos esperados & $23,08 \%(6)$ \\
Reações adversas & $11,54 \%(3)$ \\
Modo de uso & $3,84 \%(1)$ \\
Interação com outros medicamentos & $100,00 \%(26)$ \\
\hline Total &
\end{tabular}

Fonte: As autoras.

Quanto ao atendimento na farmácia da unidade de saúde, 30,77\% (n=8) delas o classificaram como inadequado, acreditando que os funcionários desse setor poderiam orientar melhor as pessoas sobre o uso dos medicamentos. Entretanto, como se pôde perceber durante a observação participante, o número de atendimentos diários e o tamanho das filas dificultavam uma orientação adequada no momento da entrega do medicamento. Além disso, a farmacêutica era responsável por mais de um centro de saúde, e quando presente, além das atividades assistenciais, desempenhava as técnico-gerenciais, que na maioria das vezes demandavam a maior parte de seu período de trabalho. Isso impossibilitava a orientação adequada dos usuários. Ademais, as farmácias não contavam com um local para atendimento individual ao usuário, e o centro de saúde nem sempre tinha consultório disponível para realização desse atendimento.

As ACSs relataram que os usuários lhes apresentavam dúvidas sobre a maneira como utilizavam os medicamentos e, muitas vezes, elas notavam que esse uso não acontecia de forma adequada. Entre os principais problemas relatados ressaltaram-se a automedicação, a não adesão ao tratamento, o uso concomitante com alimentos/álcool e o descarte inadequado de medicamentos em excesso ou vencidos. As agentes acreditavam que esse fato poderia ser justificado pela falta de orientação sobre o uso durante a consulta médica e a dispensação, além do pouco discernimento/conhecimento do usuário sobre os medicamentos e seus problemas de saúde, assim como os aspectos socioculturais que podem influenciar na utilização. Isso foi demonstrado nas seguintes falas:

Algumas pessoas tomam mais de um medicamento. É hipertenso, diabético ou que tem problema de depressão, e alguns falam que não vão tomar o remédio junto, porque pode não fazer bem (ACS 1).

Eles acham que tem que parar de tomar o remédio se 'for' tomar uma cervejinha no fim de semana. E na verdade é o contrário, eles têm que continuar, porque senão o risco é muito maior de aumentar a pressão (ACS 2). 
$\mathrm{Na}$ área tem casos da pessoa se automedicar, da vizinha falar que esse remédio foi bom para o filho, sabe? E pegar o remédio do outro. Como a amoxicilina, que às vezes sobra (ACS 2).

Também foi comum o uso indiscriminado de medicamentos como alternativa para a solução dos sintomas, como indicado nas falas seguintes:

Lá na área tem gente que, igual ela falou, tem gente que toma ibuprofeno todo dia para dor. "Que é melhor que isso e aquilo" [ACS representa usuária falando] (ACS 6).

Tem gente que toma ibuprofeno para ressaca (ACS 2).

Alguns usuários procuram o serviço de saúde com determinada queixa, entretanto não encontram resolução, como se vê a seguir:

Tem uma senhora da minha área, é outro caso diferente. Ela tem tonteira, dor de cabeça constante, ela tem labirintite, e ela toma muito dipirona. (...) Ela compra escondido para tomar, porque dizendo ela que quando toma a cabeça para de doer (ACS 2).

Ao serem indagadas por um usuário sobre o uso dos medicamentos, 53,85\% $(n=14)$ das ACSs conversam com algum profissional da sua equipe antes de fornecer respostas a ele. E ao perceberem que o usuário está tomando o medicamento de forma inadequada, 46,15\% $(\mathrm{n}=12)$ das agentes também encaminham o caso à equipe de saúde, como visto na Tabela 2.

Tabela 2

\begin{tabular}{lc}
\hline Principais condutas das ACSs nos casos de dúvidas ou uso incorreto de medicamentos & $\%(\mathbf{n})$ \\
\hline Condutas das ACSs & $53,85(14)$ \\
\hline Em caso de dúvida do usuário sobre os medicamentos & $19,23(5)$ \\
Discute o caso com profissionais da equipe de saúde da família e orienta o usuário & $19,23(5)$ \\
Orienta o usuário de acordo com seus próprios conhecimentos & $7,69(2)$ \\
Discute o caso com farmacêutico e orienta o usuário & $100,00(26)$ \\
Outra & $46,15(12)$ \\
Total & $42,31(11)$ \\
Em caso de uso incorreto de medicamentos pelo usuário & $11,54(3)$ \\
Encaminha o caso para a equipe de saúde da família & $100,00(26)$ \\
Orienta sobre o modo correto de acordo com seus próprios conhecimentos
\end{tabular}

Fonte: As autoras. 
É notável nas falas das ACSs uma insegurança no momento de orientar o usuário, bem como receio em relação à equipe, uma vez que é constante nos relatos que não é competência do agente orientar os usuários.

Ele [usuário] me pergunta alguma coisa, às vezes eu até sei falar, mas acho que não tenho a competência e tem outros profissionais que podem falar: "Quem é fulano para falar isso: ela é só ACS." [ACS simula fala de outros profissionais sobre ela.] Eu tenho que ver até onde eu posso ir (...) para mais à frente não ter que responder por uma coisa que não é sua competência. Porque já teve reunião aqui no posto, e eles [demais membros da equipe] falam assim: “Olha, isso não é competência do ACS" (ACS 4).

Quando as ACSs percebem que o usuário não está utilizando o medicamento de forma adequada, buscam apoio na equipe de saúde para que ela possa orientar da melhor maneira, como se observa nas falas seguintes:

A gente tem que trazer pra equipe, porque tem caso que a gente sabe falar que tá certo e tudo, mas como nós somos uma equipe, a gente passa o caso. Porque se tiver algo que a gente falou que não é adequado, a gente retorna à casa e fala com a pessoa de novo (ACS 1).

Eu prefiro trazer pra equipe. Prefiro trazer pra equipe e a equipe fala: “É isso mesmo." Aí, beleza! Cê volta lá e passa a informação, mas a princípio eu não passo, não (ACS 5).

Em razão das altas demandas decorrentes das atividades das ACSs, é comum o esquecimento de determinado caso que necessita de atendimento farmacêutico, como indicado a seguir:

Fácil não é, porque às vezes a gente tá muito atarefada, tem coisa que a gente acaba esquecendo mesmo no dia a dia. Por isso que às vezes a gente prefere pegar [a farmacêutica para discutir caso] no corredor. Porque quando deixa pra passar na reunião estende-se muito e mesmo se você anotou e faz os registros (ACS 2).

Até no corredor... na cozinha... lá embaixo, tomando café, nós paramos em qualquer lugar [para discutir casos com a farmacêutica] (ACS 6).

Muitas vezes, a discussão de um caso específico vivenciado em uma visita domiciliar não é realizada durante as reuniões de matriciamento com o Núcleo de Apoio à Saúde da Família (NASF) por esquecimento, como relatado pela ACS 2. Nessas situações, a ACS discute esse evento com a farmacêutica 
em qualquer local que a encontrar no centro de saúde, como reforçado pela ACS 6. Em outras situações, o farmacêutico se tornou a referência no acompanhamento dos usuários em tratamento de tuberculose:

Nos casos de TBC [tuberculose] que eu tive, ela [farmacêutica] ajudou muito. Toda vez que precisou, ela chamou a pessoa aqui, conversou, orientou sobre o medicamento (ACS 4).

Antes era na equipe. Agora eu já vou diretamente nela [farmacêutica], já agenda consulta, já marca o dia do remédio. Ela já fala o dia que vai acabar. Interajo mais com ela do que com a equipe (ACS 8).

As falas das ACSs revelam que, nesse cenário, o papel do farmacêutico limita-se ao apoio à equipe no manejo do cuidado, com foco principal no tratamento medicamentoso para auxiliar na adesão. Além disso, é pouco expressiva a procura pelo farmacêutico para discutir dúvidas provenientes do uso de medicamentos, bem como o encaminhamento de usuários para atendimento clínico por esse profissional (Tabela 2).

\section{Necessidade de apoio aos usuários e aos ACSs nas dificuldades enfrentadas com o uso de medicamentos}

O trabalho do ACS compreende o intercâmbio entre dois polos e envolve complexidade, uma vez que ele adentra nos domicílios, recebe as queixas da população e se compromete a dar respostas e encaminhar soluções para os problemas encontrados. Ao mesmo tempo, relaciona-se com a equipe de saúde, age segundo as possibilidades e os limites desta e do próprio sistema de saúde. A integração entre o trabalho do ACS e o da equipe é essencial para o compartilhamento de saberes e para manutenção e qualificação da assistência prestada (Galavote et al., 2011; Peres et al., 2011). A proximidade e o vínculo dos usuários com o ACS facilitam a troca de informações entre eles, o que os leva a aproveitar o momento da visita para esclarecer questões sobre seu tratamento, sendo comum o relato de dúvidas sobre medicamentos.

A frequência das dúvidas dos usuários sobre medicamentos observada no estudo aqui apresentado, segundo a percepção das ACSs, pode relacionarse à qualidade do atendimento na farmácia. A falta de informações sobre os medicamentos ou a não compreensão delas pelos usuários pode levar ao seu uso inadequado, prejudicar a efetividade e a segurança do tratamento e resultar em morbimortalidade relacionada à farmacoterapia (Ernst e Grizzle, 2001). Por isso, a educação do usuário sobre seus medicamentos é fundamental para o sucesso do tratamento. A ausência de processos educativos efetivos 
pode trazer consequências como não adesão ao tratamento, confusão entre manifestações da doença e efeitos adversos dos medicamentos e incentivo à automedicação, entre outras que podem piorar o estado de saúde do usuário (Oenning, Oliveira e Blatt, 2011).

Segundo a Política Nacional de Atenção Básica (PNAB), é atribuição do agente estabelecer contato permanente com as famílias, com o desenvolvimento de ações educativas visando à promoção da saúde, à prevenção das doenças e ao acompanhamento das pessoas com problemas de saúde. Portanto, o ACS não erra em orientar os usuários, porém a informação deve estar correta, e para que isso aconteça se faz necessário que esse profissional esteja capacitado e tenha suporte dos demais profissionais de saúde(Brasil, 2012).

Nas dinâmicas da equipe de saúde, percebe-se a relação de hierarquia de conhecimento, sendo o detentor do poder aquele que possuir maior nível de escolaridade e exercer profissão com maior reconhecimento social. Em vista disso, o ACS percebe-se como o profissional que detém menos poder. Nesse caso, é importante reconhecer o trabalho realizado pelo outro trabalhador, caracterizando-o como parte constitutiva de ações coletivas em saúde. Portanto, para que essa realidade seja modificada, é imprescindível a interação social entre os trabalhadores para permitir maior autonomia e criatividade dos agentes e maior integração da equipe (Galavote et al., 2011; Peres et al., 2011).

Uma atenção primária com qualidade e efetiva deve ser caracterizada como o nível de assistência primordial para a resolução da maioria dos problemas de saúde da comunidade, em tempo conveniente, com acessibilidade e garantia da continuidade do cuidado(Almeida, Fausto e Giovanella, 2011). Para que o atendimento ao usuário seja efetivo, deve-se ressaltar a importância do diálogo e do acolhimento às demandas e necessidades da população atendida. Além disso, os resultados positivos de uma intervenção dependem dos conhecimentos técnicos dos profissionais de saúde, da capacidade de escuta e do estabelecimento de confiança com a população, essenciais para o desempenho de suas atribuições com qualidade e efetividade (Almeida, Fausto e Giovanella, 2011; Campos et al., 2014). Nesse sentido, a presença do farmacêutico na APS, por meio do Nasf, é um avanço no cuidado ao usuário, uma vez que sua atuação integrada às equipes de saúde da família e a prestação de apoio especializado, assistencial e/ou técnico-pedagógico podem contribuir para o aumento da qualidade da assistência farmacêutica e da resolutividade das ações de saúde que visem à promoção, à prevenção, à reabilitação e à educação em saúde(Brasil, 2009). A proximidade do ACS com o farmacêutico pode facilitar a resolução do problema do usuário de medicamentos e auxilia na promoção do uso correto e na educação do usuário sobre a farmacoterapia. Consequentemente, o tratamento tem mais chances de ser efetivo e o usuário de se tornar mais capacitado para lidar com possíveis problemas decorrentes 
do uso de medicamentos, contribuindo para a adesão ao tratamento (Costa, Rabelo e Lima, 2014).

Não se pretende propor que o ACS se responsabilize pela orientação do uso correto de medicamentos, mas sim capacitá-lo a identificar problemas decorrentes de sua utilização, fazendo a conexão entre a necessidade do usuário e os profissionais capazes de atendê-la.

É importante que a assistência farmacêutica seja exercida de forma satisfatória, ou seja, que o farmacêutico consiga desenvolver não só atividades relacionadas ao âmbito técnico-gerencial mas também assistenciais, de maneira a promover o uso adequado do medicamento - o que é primordial para que o tratamento seja efetivo e diminuam-se os riscos, com consequente impacto na resolução de ações em saúde (Mendes et al., 2014). Entretanto, a literatura aponta que, ao exercer suas funções na lógica do NASF (Silva, 2015), nem sempre isso é possível no cotidiano de trabalho, devido à maior demanda das funções gerenciais, que limitam a plena execução das funções assistenciais.

Como observado no trabalho de Nakamura e Leite (2016), os farmacêuticos do Nasf que desenvolvem suas atividades em várias unidades de saúde têm dificuldades com a criação de vínculos e referência com o serviço. Além disso, segundo essas autoras, não há entendimento e compreensão por parte da equipe de saúde sobre o verdadeiro papel do farmacêutico no cuidado à saúde, por isso muitas vezes os casos clínicos não são direcionados a esse profissional durante o apoio matricial. Dessa forma, além de capacitar o ACS para identificar os potenciais problemas com o uso de medicamentos, há que se cuidar de haver profissionais preparados e com disponibilidade de tempo adequada para atender a essa demanda.

A baixa procura das ACSs pelo farmacêutico no estudo aqui apresentado pode estar relacionada à imagem desse profissional socialmente construída desde a industrialização dos medicamentos, em que ele se distancia do contato direto com os usuários (Saturnino et al., 2012). Em estudos realizados por Nakamura e Leite $(2015,2016)$, verificou-se que o farmacêutico dedica a maior parte do seu tempo de trabalho às atividades de gestão, enquanto as atividades assistenciais ficam restritas às necessidades que são observadas pela equipe de saúde ou pelos próprios farmacêuticos. Além disso, as atividades de assistência dependem das habilidades de criatividade, disponibilidade e interesse de cada profissional farmacêutico. Isso pode ter relação com a falta de reconhecimento do papel do farmacêutico como profissional de saúde pela equipe, o que pode limitar o cuidado à saúde dos usuários e o trabalho do Nasf no manejo dos casos. Assim, faz-se necessário repensar a atuação clínica do farmacêutico na atenção primária e avançar em seu desenvolvimento, tanto no município onde o estudo foi desenvolvido quanto em outros cenários, ampliando suas responsabilidades no cuidado ao usuário juntamente com a equipe de saúde, a exemplo de outras experiências de sucesso no Brasil (Brasil, 
2014). Nessa perspectiva de ampliação da prática clínica dos farmacêuticos na atenção básica, sua parceria com os ACSs pode potencializar as ações dessas duas categorias profissionais, ao favorecer práticas que possam estabelecer o intercâmbio entre o conhecimento técnico-científico do profissional e o saber popular dos usuários, propiciando um cuidado mais qualificado das famílias (Kauling et al., 2013; Prata et al., 2012).

\section{Considerações finais}

Dúvidas e problemas decorrentes da utilização de medicamentos se apresentam cotidianamente aos ACSs. Algumas intervenções são feitas por esses profissionais diretamente com os usuários, prevalecendo, porém, o encaminhamento à equipe de saúde. Constatou-se que os agentes não se sentem competentes para exercer seu papel de promotores do uso correto de medicamentos, como está estabelecido nas diretrizes do Ministério da Saúde (Brasil, 2006). Assim, é preciso reconhecer seu papel na atenção à saúde e, como consequência, capacitá-los para que detenham conhecimento suficiente para identificar problemas que necessitem de intervenções, realizar ações e encaminhamentos necessários, possibilitando que seja oferecido cuidado com qualidade à população.

O farmacêutico, mesmo participando da equipe de apoio matricial à equipe de saúde, é pouco demandado pelos ACSs para sanar dúvidas ou realizar atendimento aos usuários de medicamentos. Torna-se necessária mudança na prática desse profissional, com foco no cuidado direto ao paciente, de forma que os demais profissionais passem a (re)conhecer seu potencial na redução da morbimortalidade relacionada ao uso de medicamentos. Além disso, o estabelecimento de uma parceria efetiva entre a equipe de saúde, incluindo os ACSs, e farmacêutico pode contribuir para ações que melhorem os resultados da utilização de medicamentos pela população. 


\section{Colaboradoras}

Maria do Carmo Vilas Boas Sousa elaborou o projeto de pesquisa, desempenhou as atividades práticas, coleta e análise dos dados, revisão da literatura, concepção, desenvolvimento e redação final do artigo. Cynthia Pimenta dos Santos colaborou na análise crítica e na redação final. Simone de Araújo Medina Mendonça auxiliou no desenvolvimento do projeto e contribuiu na análise crítica e redação final do artigo. Não houve financiamento e não há conflito de interesses.

Resumen El agente comunitario de salud representa um elemento importante en la gestión de la información sobre el uso de medicamentos en la atención primaria, mejorando la interacción del equipo de salud con la comunidad. El objetivo de este estudio fue revelar cómo el uso de medicamentos está presente en la rutina de los agentes y entender las relaciones que se establecen entre agentes, usuarios y equipo de salud. Se trata de un estudio cuantitativo y cualitativo, utilizando la observación participante, entrevista semi-estructurada y grupos focales. Lo studio fue desenvolvido en tres unidades básicas de salud en um municipio de Minas Gerais, Brasil, en el período de marzo de 2013 a febrero de 2015. Los resultados muestran que los agentes se relacionan constantemente con usuarios que experimentan problemas con el uso de medicamentos y que tienen dudas acerca de la indicación de medicamentos, los efectos esperados, las reacciones adversas, entre otros. Los agentes realizan algunas intervenciones directamente con el usuario, no obstante prefieren referir los casos para el equipo de salud. Algunos agentes se refieren al farmacéutico como profesional de referencia con respecto al uso de medicamentos, sin embargo, la demanda de estos profesionales por los sujetos de este estudio fue baja. Se resalta la importancia de la formación de los agentes sobre el uso de medicamentos, para darles instrumentos en el reconocimiento de situaciones inadecuadas, el desarrollo de intervenciones con el equipo de salud y el seguimiento de los usuarios. Palabras clave uso de medicamento; agentes comunitarios de salud; investigación curativa. 


\section{Notas}

${ }^{1}$ Universidade Federal de Minas Gerais, Programa de Pós-Graduação em Medicamentos e Assistência Farmacêutica, Faculdade de Farmácia, Belo Horizonte, Minas Gerais, Brasil. $<$ mariavilassboas@gmail.com>

Correspondência: Faculdade de Farmácia, Universidade Federal de Minas Gerais, Av. Presidente Antônio Carlos, 6.627, CEP 31270-901, São Luiz, Belo Horizonte, Minas Gerais, Brasil.

${ }^{2}$ Secretaria Municipal de Saúde de Belo Horizonte, Núcleo de Apoio à Saúde da Família, Belo Horizonte, Minas Gerais, Brasil.

<cynthiaspimenta@gmail.com>

${ }^{3}$ Universidade Federal de Juiz de Fora, Campus Governador Valadares. Governador Valadares, Minas Gerais, Brasil.

<simoneamm@gmail.com>

\section{Referências}

ALMEIDA, Patty F.; FAUSTO, Márcia C. R.; GIOVANELLA, Lígia. Fortalecimento da atenção primária à saúde: estratégia para potencializar a coordenação dos cuidados. Revista Panamericana de Salud Publica, Washington, v. 29, n. 2, p. 84-95, 2011.

BALCAZAR, Hector et al. Community health workers can be a public health force for change in the United States: three actions for a new paradigm. American Journal of Public Health, Washington, v. 101, n. 12, p. 2.199-2.203, 2011.

BRASIL. Ministério da Saúde. Secretaria de Ciência, Tecnologia e Insumos Estratégicos. o trabalho dos agentes comunitários de saúde na promoção do uso correto de medicamentos. Brasília: Ministério da Saúde, 2006. 72 p. (Série F. Comunicação e Educação em Saúde).

BRASIL. Ministério da Saúde. Secretaria de Atenção à Saúde. Departamento de Atenção Básica. O trabalho do agente comunitário de saúde. Brasília: Ministério da Saúde, 2009. 84 p. (Série F. Comunicação e Educação em Saúde).

BRASIL. Ministério da Saúde. Secretaria de Atenção à Saúde. Departamento de Atenção Básica. Política Nacional de Atenção Básica. Brasília: Ministério da Saúde, 2012. 110 p. (Série E. Legislação em Saúde).

BRASIL. Ministério da Saúde. Secretaria de Ciência, Tecnologia e Insumos Estratégicos. Departamento de Assistência Farmacêutica e Insumos Estratégicos. Serviços farmacêuticos na atenção básica à saúde. Brasília: Ministério da Saúde, 2014. 108 p. (Cuidado farmacêutico na atenção básica; caderno 1).

BRITO, Rosineide S.; FERREIRA, Nathaly E. M. S.; SANTOS, Danyelle L. A. Atividades dos agentes comunitários de saúde no âmbito da Estratégia Saúde da Família: revisão integrativa da literatura. Saúde \& Transformação Social, Florianópolis, v. 5, n. 1, p. 16-21, 2014. 
CAMPOS, Rosana T. O. et al. Avaliação da qualidade do acesso na atenção primária de uma grande cidade brasileira na perspectiva dos usuários. Saúde em Debate, Rio de Janeiro, v. 38, n. esp., p. 252-264, 2014.

COLLINSWORTH, Ashley et al. Community health workers in primary care practice: redesigning health care delivery systems to extend and improve diabetes care in underserved populations. Health Promotion Practice, Washington, v. 15, supl. 2, p. 51-61, nov. 2014.

COSTA, Evandro M.; RABELO, Aneide R. M.; LIMA, José G. Avaliação do papel do farmacêutico nas ações de promoção da saúde e prevenção de agravos na atenção primária. Revista de Ciências Farmacêuticas Básica e Aplicada, Araraquara, v. 35, n. 1, p. 81-88, 2014.

COSTA, Simone M. et al. Agente comunitário de saúde: elemento nuclear das ações em saúde. Ciência \& Saúde Coletiva, Rio de Janeiro, v. 18, n. 7, p. 2.147-2.156, 2013.

ERNST, Frank R.; GRIZZLE, Amy J. Drugrelated morbidity and mortality: updating the cost-of-illness model. Journal of the American Pharmacists Association, Washington, v. 41, n. 2, p. 192-199, 2001.

GALAVOTE, Heletícia S. et al. Desvendando os processos de trabalho do agente comunitário de saúde nos cenários revelados na Estratégia Saúde da Família no município de Vitória (ES, Brasil). Ciência \& Saúde Coletiva, Rio de Janeiro, v. 16, n. 1, p. 231-240, 2011.

GOULART, Letícia S. et al. Consumo de medicamentos por idosos de uma unidade básica de saúde de Rondonópolis/MT. Estudos Interdisciplinares sobre o Envelhecimento, Porto Alegre, v. 19, n. 1, p. 79-94, 2014.

HEER, Hendrik D. et al. A path analysis of a randomized promotora de salud cardiovascular disease-prevention trial among at-risk Hispanic adults. Health Education \& Behavior, Thousand Oaks, v. 39, n. 1, p. 77-86, 2012.
KAULING, Greice P. et al. Utilização de medicamentos: limites e possibilidades das orientações dos agentes comunitários de saúde às famílias. O Mundo da Saúde, São Paulo, n. 37, v. 1, p. 44-55, 2013.

KRANTZ, Mori J. et al. Effectiveness of a community health worker cardiovascular risk reduction program in public health and health care settings. American Journal of Public Health, Washington, v. 103, n. 1, p. 19-27, 2013.

LASTE, Gabriela et al. Papel do agente comunitário de saúde no controle do estoque domiciliar de medicamentos em comunidades atendidas pela Estratégia Saúde da Família. Ciência \& Saúde Coletiva, Rio de Janeiro, v. 17, n. 5, p. 1.305-1.312, maio 2012.

MARODIN, Gabriela et al. Agentes comunitários de saúde, idosos e PET-Saúde: uso e cuidados com medicamentos. Revista Contexto \& Saúde, Ijuí, v. 10, n. 20, p. 635-642, 2011.

MASTROIANNI, Patrícia C. et al. Estoque doméstico e uso de medicamentos em uma população cadastrada na Estratégia Saúde da Família no Brasil. Revista Panamericana de Salud Publica, Washington, v. 29, n. 5, p. 358-364, 2011.

MEDEIROS, Eloá F. F. et al. Intervenção interdisciplinar enquanto estratégia para o uso racional de medicamentos em idosos. Ciência \& Saúde Coletiva, Rio de Janeiro, v. 16, n. 7, p. 3.139-3.149, 2011.

MENDES, Luiz V. et al. Disponibilidade de medicamentos nas unidades básicas de saúde e fatores relacionados: uma abordagem transversal. Saúde em Debate, Rio de Janeiro, v. 38, n. esp., p. 109-123, out. 2014.

NAKAMURA, Carina A.; LEITE, Silvana N. Pharmaceutical services in family health support team: the Brazilian experience. Latin American Journal of Pharmacy, Buenos Aires, v. 34, n. 3, 598-601, 2015. 
NAKAMURA, Carina A.; LEITE, Silvana N. A construção do processo de trabalho no Núcleo de Apoio à Saúde da Família: a experiência dos farmacêuticos em um município do Sul do Brasil. Ciência \& Saúde Coletiva, Rio de Janeiro, v. 21, n. 5, p. 1.565-1.572, 2016.

NUNES, Carla C.; AMADOR, Tânia A.; HEINECK, Isabela. O medicamento na rotina de trabalho dos agentes comunitários de saúde da Unidade Básica de Saúde Santa Cecília, em Porto Alegre, RS, Brasil. Saúde e Sociedade, São Paulo, v. 17, n. 1, p. 85-94, 2008.

OENNING, Diony; OLIVEIRA, Bruna V.; BLATT, Carine R. Conhecimento dos pacientes sobre os medicamentos prescritos após consulta médica e dispensação. Ciência \& Saúde Coletiva, Rio de Janeiro, v. 16, n. 7, p. 3.277-3.283, 2011.

PERES, Cássia R. F. B. et al. O agente comunitário de saúde frente ao processo de trabalho em equipe: facilidades e dificuldades. Revista da Escola de Enfermagem da USP, São Paulo, v. 45, n. 4, p. 905-911, 2011.

PRATA, Pollyana B. A. et al. Atenção farmacêutica e a humanização da assistência: lições aprendidas na promoção da adesão de usuários aos cuidados terapêuticos nas condições crônicas. O Mundo da Saúde, São Paulo, v. 36, n. 3, p. 526-530, 2012.

SATURNINO, Luciana T. M. et al. Farmacêutico: um profissional em busca de sua identidade. Revista Brasileira de Farmácia, Rio de Janeiro, v. 93, n. 1, p. 10-16, 2012.

SILVA, Danielle F. Construção de um serviço de gerenciamento da terapia medicamentosa na atenção primária à saúde no SUS: um projeto de extensão universitária. 2015. 104f. Dissertação (Mestrado em Medicamentos e Assistência Farmacêutica) - Faculdade de Farmácia, Universidade Federal de Minas Gerais, Belo Horizonte, 2015.

SPENCER, Michael S. et al. Effectiveness of a community health worker intervention among African American and Latino adults with type 2 diabetes: a randomized controlled trial. American Journal of Public Health, Washington, v. 101, n. 12, p. 2.253-2.260, 2011.

Recebido em 16/10/2016. Aprovado em 11/07/2017. 